\title{
Acute Pancreatitis Induced Splenic Vein Thrombosis
}

\author{
Chidinma Ejikeme ${ }^{1}$, Sherif Elkattawy ${ }^{1}$, Fisayo Kayode-Ajala ${ }^{2}$, Abraheim Al-nasseri ${ }^{3}$, Arun Naik ${ }^{4}$ \\ 1. Internal Medicine, Rutgers New Jersey Medical School/Trinitas Regional Medical Center, Elizabeth, USA 2. Internal \\ Medicine, Trinitas Regional Medical Center, Elizabeth, USA 3. Internal Medicine, St. George's University School of \\ Medicine, Elizabeth, USA 4. Gastroenterology, Trinitas Regional Medical Center, Elizabeth, USA
}

Corresponding author: Arun Naik, anaik@gmail.com

\begin{abstract}
Acute inflammation of the pancreas, known as pancreatitis, can result in many complications ranging from acute distress respiratory syndrome to pancreatic necrosis. A relatively common vascular complication of pancreatitis is splenic vein thrombosis (SVT) due to intimal inflammation leading to platelet aggregation and thrombosis. The management of SVT with regard to anticoagulation (AC) might appear to be perplexing at first given the recommendation to withhold any sort of AC. Research studies have shown that these patients have an increased risk of gastrointestinal bleeding without AC. In this report, we discuss a case of hypertriglyceridemia-induced pancreatitis. During hospitalization, our patient complained of worsening abdominal pain with objective fevers and leukocytosis in which CT scan of the abdomen was significant for hemorrhagic pancreatitis with necrosis, acute SVT, and splenomegaly. The patient was managed conservatively with IV fluids, pain relief medications, and antibiotics.
\end{abstract}

Review began 05/31/2021 Review ended 06/03/2021 Published 06/17/2021

(c) Copyright 2021 Ejikeme et al. This is an open access article distributed under the terms of the Creative Commons Attribution License CC-BY 4.0., which permits unrestricted use, distribution, and reproduction in any medium, provided the original author and source are credited.
Categories: Internal Medicine, Medical Education, Gastroenterology

Keywords: pancreatitis, splenic vein thrombosis, complications, pancreatic exocrine insufficiency, inflammation

\section{Introduction}

Pancreatitis by definition refers to an acute or chronic inflammation of the pancreas. Chronic pancreatitis can result in permanent structural changes which can lead to exocrine and endocrine dysfunction [1]. Pancreatitis complications include pancreatic duct dysfunction, obstruction, and vascular complications [23]. We present a case of a 28 -year-old male with a past medical history (PMH) of type I diabetes mellitus, and alcohol abuse who developed acute splenic vein thrombosis (SVT) secondary to interstitial edematous pancreatitis. SVT represents one of the vascular complications of chronic pancreatitis [2]. Patients with this complication typically are asymptomatic, however, symptomatic patients usually present with gastric varices and evidence of splenomegaly on physical examination [3]. Our case will highlight the development of SVT post-pancreatitis inflammation.

\section{Case Presentation}

A 28-year-old male with a past medical history (PMH) of type I diabetes mellitus and alcohol abuse presented to the emergency department (ED) with complaints of abdominal pain. His symptoms started on awakening that morning. He described it as a sudden onset epigastric pain, rated 10/10 and nonradiating. The patient also became nauseous and had episodes of non-bilious, non-bloody vomiting, which prompted his ED visit. The patient denied headaches, dizziness, chest pain, shortness of breath, diarrhea, dysuria, or neurological changes. In the $\mathrm{ED}$, vital signs were remarkable for a temperature of $98.9^{\circ} \mathrm{F}$, heart rate of $110 \mathrm{bpm}$, respiratory rate of 15 breaths/min, and blood pressure (bp) of 160/111 $\mathrm{mmHg}$. Labs were remarkable for white cell count $20.3 \mathrm{k} / \mathrm{uL}$, hemoglobin $17.4 \mathrm{~g} / \mathrm{dL}$, and serum lipase level $665 \mathrm{u} / \mathrm{L}$. The lipid panel revealed a total cholesterol level of $1154 \mathrm{mg} / \mathrm{dL}$, high density lipoprotein (HDL) of $23 \mathrm{mg} / \mathrm{dl}$, with significantly elevated low density lipoprotein (LDL) and triglyceride level, such that it was too high to calculate due to lipemia. A CT scan of the abdomen without contrast revealed acute interstitial edematous pancreatitis primarily involving the pancreas body and tail. There was also acute peripancreatic fluid collection measuring $7 \mathrm{~cm} \times 4 \mathrm{~cm} \times 5 \mathrm{~cm}$ as seen in Figure 1. 


\section{Cureus}

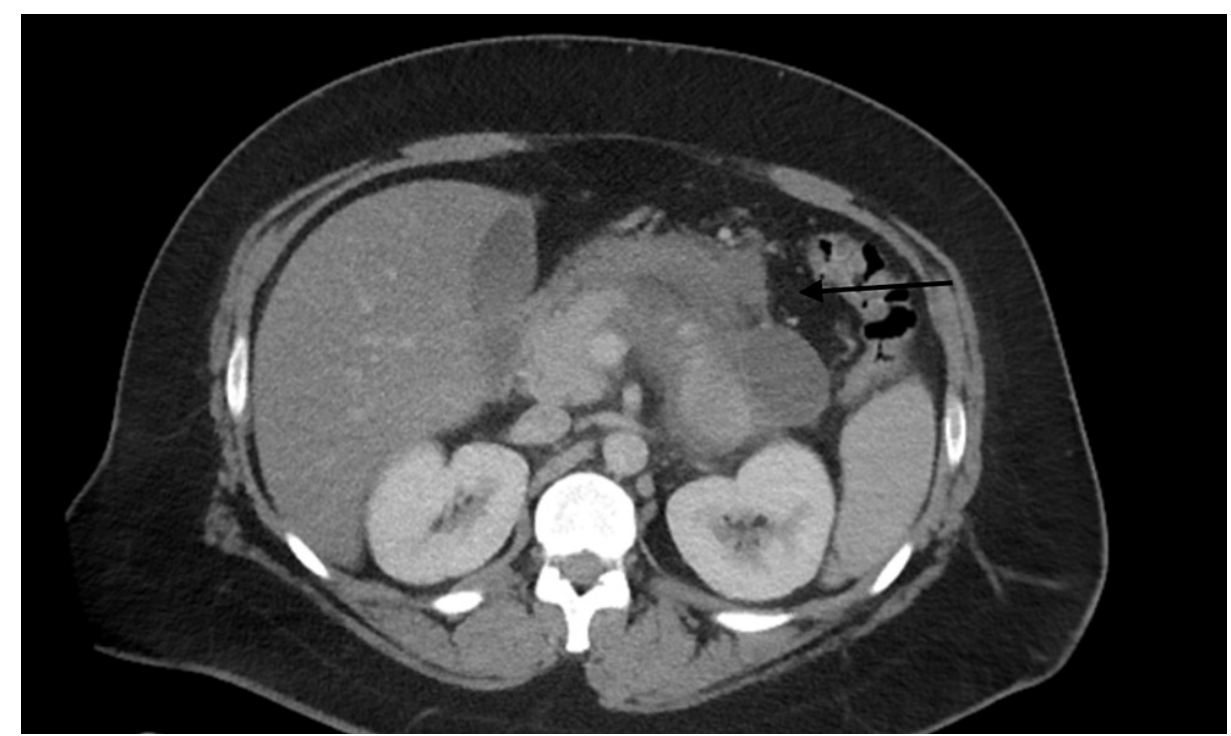

FIGURE 1: CT scan of the abdomen without contrast revealed acute interstitial edematous pancreatitis primarily involving the pancreas body and tail.

Of note, the patient was admitted in 2019 with similar symptoms where he was diagnosed with hypertriglyceridemia-induced pancreatitis and was discharged on atorvastatin and gemfibrozil. During this evaluation, the patient stated that he is non-compliant with medications and that he now drinks an average of four cans of beer twice weekly.

Given hypertriglyceridemia, the patient was placed on strict nil per os (NPO) and transferred to the ICU for further management. IV fluid and insulin infusion were initiated for the management of recurrent hypertriglyceridemia pancreatitis. Frequent blood work was performed to monitor lipid panel and potassium level while on insulin infusion. About three days following treatment, the patient's symptoms started to improve on the current treatment. Repeat lipid panel showed an improvement of triglyceride level at 470 $\mathrm{mg} / \mathrm{dL}$, LDL of $177 \mathrm{mg} / \mathrm{dL}$, and total cholesterol of $284 \mathrm{mg} / \mathrm{dL}$. He reported having minimal to no abdominal pain; hence he was advanced to a clear liquid diet. He was placed on a low-fat diet, atorvastatin, and gemfibrozil for better lipemic control. While on current management, the patient's abdominal pain started to worsen again. He began to have multiple episodes of fever. His white cell count started to increase significantly, and his hemoglobin level decreased to $12.7 \mathrm{~g} / \mathrm{dL}$. A repeat CT abdomen was performed, which revealed an extensive hyperdense peripancreatic fluid with findings suggestive of hemorrhagic pancreatitis, with acute SVT and splenomegaly as seen in Figure 2. There were also new areas of non-enhancement within the pancreatic tail compatible with necrosis as seen in Figure 3. 


\section{Cureus}

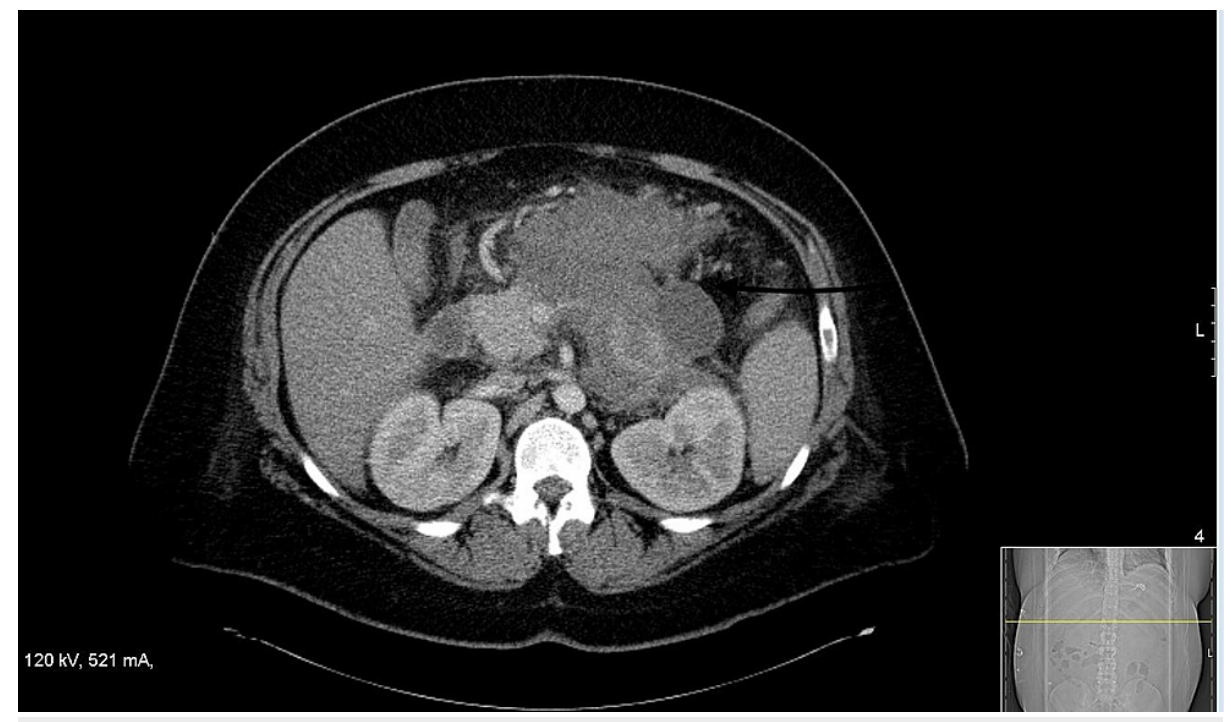

FIGURE 2: CT scan of the abdomen significant for extensive hyperdense peripancreatic fluid, consistent with hemorrhagic pancreatitis.

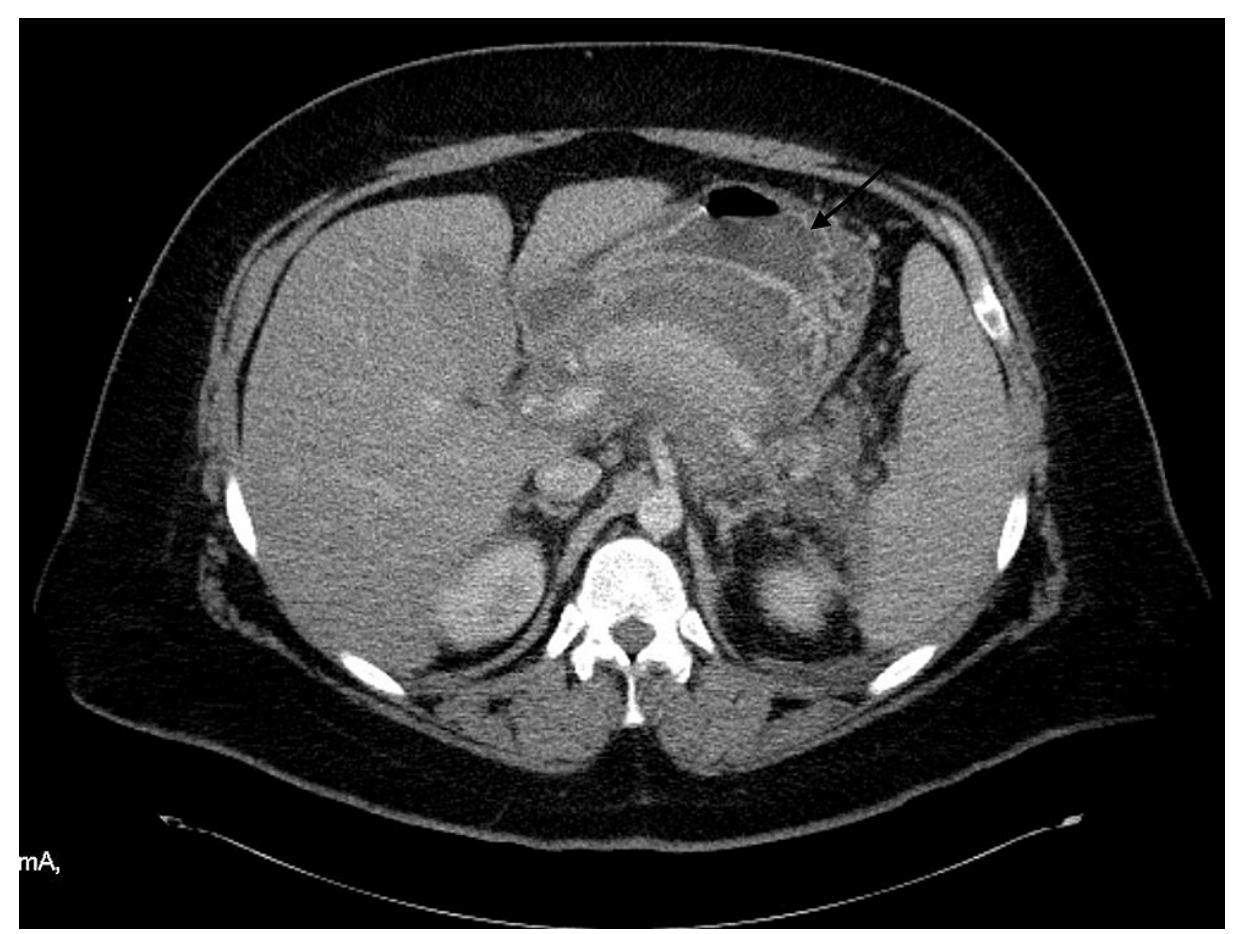

FIGURE 3: CT scan of the abdomen shows new areas of nonenhancement within the pancreatic tail, compatible with necrosis.

The patient was started on meropenem, and hemoglobin levels were monitored frequently. The hepatobiliary surgery team was consulted, who recommended supportive management with antibiotics, IV fluid, and to advance diet as tolerated. Blood cultures were performed, which were negative. The patient's symptoms eventually improved and his hemoglobin levels stabilized. He was gradually restarted on a consistent carbohydrate diet without any new complications. The patient was discharged with lipidlowering medications and scheduled for an outpatient appointment.

\section{Discussion}

The management of our patient's pancreatitis-induced splenic vein thrombosis (PIVST) was mostly 
supportive measures with significant improvement of symptoms. Inflammation of the pancreas may lead to inflammation of the underlying splenic vein, leading to thrombus formation and other pathological sequelae (i.e., left-sided portal hypertension and gastric varices) [4-5]. Since acute inflammation encompasses the capacity to resolve, treatment of the underlying pancreatic inflammation eventually eliminates complications associated with splenic vein inflammation [6-9]. An understanding of the pathophysiology involved in PISVT supports our approach in management and simplifies the treatment strategy for select patients (young patients with gradual improvement of acute pancreatitis (AP) under standard therapy, and have no history of bleeding diatheses).

In addition to the pathophysiology, clinical research also supports standard AP treatment as a means of managing PIVST in select patients [5]. Gonzelez et al. reported that no significant differences were observed in AP-induced splanchnic vein recanalization, irrespective of whether or not they received anticoagulation (AC) therapy $(\mathrm{p}=0.076)$. Furthermore, the study concluded that the self-limited treatment for AP with splanchnic thrombosis could be a result of the self-limiting resolution of AP and/or drainage of adjacent collections [1]. AC may also be a relative contradiction when treating PIVST, as a cohort study by Anderson et al. concluded that AC significantly increased the risk for gastrointestinal (GI) bleeding $(\mathrm{p}=$ 0.032).2 PIVST already holds a $12.3 \%$ increased risk of GI bleeding without AC, this becomes especially useful for practicing evidence-based medicine (i.e., assessing the risk of hemorrhage during pancreatic surgery in a patient diagnosed with PIVST) $[3,6]$. In support of utilizing CT imaging to monitor our patient's disease course, many researchers have proven the CT scan to be statistically significant in the accurate prediction of pancreatitis severity and prevalence of SVT [4, 7-8]. This becomes especially useful when determining whether or not self-limited management of PISVT is clinically useful in selective patients.

As seen in our case, PISVT is a clinically relevant complication of AP and it is imperative to delineate the management strategy for these patients. Although several complications may arise in AP, it is imperative to differentiate between patients that only require a standard approach to care, from those that require advanced medical management [i.e., acute respiratory distress syndrome (ARDS) secondary to AP]. However, the treatment of PISVT must not be oversimplified, as standard management entails critical monitoring of the disease course during hospitalization. Additionally, some reports have determined splenomegaly as an asymptomatic sign of PIVST that may require splenectomy prior to pancreatic surgery $[3,10]$. Further studies are needed to support the use of self-limited treatment for PISVT while stratifying patient demographics (including age, race, frequency of AP, and comorbidities). In addition, studies that measure the severity of portal vein thrombosis associated with PISVT, in comparison to PISVT alone, may be clinically significant in determining prognosis and providing more insight into the risks and benefits of treatment.

\section{Conclusions}

The incidence of PISVT is estimated to be about $12 \%$ in the general population. In our case, our patient's symptoms were self-limiting and resolved with standard treatment for pancreatitis. Further research is still needed to ascertain the benefits of self-limited treatment in addition to AC in PISVT patients.

\section{Additional Information}

\section{Disclosures}

Human subjects: Consent was obtained or waived by all participants in this study. Conflicts of interest: In compliance with the ICMJE uniform disclosure form, all authors declare the following: Payment/services info: All authors have declared that no financial support was received from any organization for the submitted work. Financial relationships: All authors have declared that they have no financial relationships at present or within the previous three years with any organizations that might have an interest in the submitted work. Other relationships: All authors have declared that there are no other relationships or activities that could appear to have influenced the submitted work.

\section{References}

1. Gonzelez HJ, Sahay SJ, Samadi B, Davidson BR, Rahman SH: Splanchnic vein thrombosis in severe acute pancreatitis: a 2-year, single-institution experience. HPB (Oxford). 2011, 13:860-864. 10.1111/j.14772574.2011.00392.x

2. Anderson W, Niccum B, Chitnavis M, Uppal D, Hays AR: MD outcomes of anticoagulation for portal and/or splenic vein thrombosis in setting of acute pancreatitis. Am J Gastroenterol. 2017, 112:S6.

3. Butler JR, Eckert GJ, Zyromski NJ, Leonardi MJ, Lillemoe KD, Howard TJ: Natural history of pancreatitisinduced splenic vein thrombosis: a systematic review and meta-analysis of its incidence and rate of gastrointestinal bleeding. HPB (Oxford). 2011, 13:839-845. 10.1111/j.1477-2574.2011.00375.x

4. Mortelé KJ, Mergo PJ, Taylor HM, Ernst MD, Ros PR: Splenic and perisplenic involvement in acute pancreatitis: determination of prevalence and morphologic helical CT features. J Comput Assist Tomogr. 2001, 25:50-54. 10.1097/00004728-200101000-00009

5. Gündüz E, Dursun R, İçer M, Zengin Y, Güloğlu C: Acute pancreatitis and splenic vein thrombosis due to hypertriglyceridemia. Case Rep Gastrointest Med. 2015, 2015:729510. 10.1155/2015/729510

6. Yadav D, Lowenfels AB: The epidemiology of pancreatitis and pancreatic cancer. Gastroenterology. 2013, 144:1252-1261. 10.1053/j.gastro.2013.01.068

7. Agarwal AK, Raj Kumar K, Agarwal S, Singh S: Significance of splenic vein thrombosis in chronic 


\section{Cureus}

pancreatitis. Am J Surg. 2008, 196:149-154. 10.1016/j.amjsurg.2007.07.039

8. Uy PPD, Francisco DM, Trivedi A, O'Loughlin M, Wu GY: Vascular diseases of the spleen: a review. J Clin Transl Hepatol. 2017, 5:152-164. 10.14218/JCTH.2016.00062

9. Freire MO, Van Dyke TE: Natural resolution of inflammation. Periodontol 2000. 2013, 63:149-164. 10.1111/prd.12034

10. Freedman S, Forsmark C: Overview of the complications of chronic pancreatitis . UpToDate. 2020, 